\title{
Accuracy and convergence of iteratively solved Monte Carlo codes for simulations in the plasma edge of nuclear fusion reactors
}

\author{
Kristel Ghoos $^{1^{*}}$ | Giovanni Samaey ${ }^{2}$ | Martine Baelmans ${ }^{1}$
}

\author{
${ }^{1}$ Department of Mechanical Engineering, KU \\ Leuven, Leuven, Belgium \\ ${ }^{2}$ Department of Computer Science, KU Leuven, \\ Leuven, Belgium \\ *Correspondence \\ Kristel Ghoos, Department of Mechanical \\ Engineering, KU Leuven, Celestijnenlaan 300A, \\ 3001 Leuven, Belgium. \\ Email: kristel.ghoos@kuleuven.be \\ Funding Information \\ This research was supported by the Flanders \\ Innovation and Entrepreneurship, IWT.141064.
}

Iteratively solved Monte Carlo (MC) codes are frequently used for plasma edge simulations. However, their accuracy and convergence assessment are still unresolved issues. In analogy with the error classification recently developed for coupled finite-volume/Monte Carlo (FV-MC) codes, we define different error contributions and analyse them separately in a simplified non-linear MC code. Three iterative procedures are examined: Random Noise $(\mathrm{RN})$, where different seeds are used in each iteration; Correlated Sampling, where particle trajectories remain correlated between iterations; and Robbins Monro, where averaging is used during the simulation. We show that, as in FV-MC codes, $\mathrm{RN}$ is the most efficient iterative procedure provided averaging is used to decrease the statistical error. In addition, we conclude that the accuracy can be assessed using the same techniques as in FV-MC codes.

\section{KEYWORDS}

accuracy, convergence, Monte Carlo, numerical errors

\section{1 | INTRODUCTION}

Monte Carlo (MC) procedures are often used in the plasma edge to solve non-linear problems such as scrape-off layer simulations with B2-EIRENE or EMC3-EIRENE. They are also used to assess neutral particle transport in high collisional regions further away from the plasma, e.g. to assess the performance of pumps or neutral densities in the outer regions of the reactor. However, the assessment of convergence and numerical errors is often missing. As such, the coupled MC-MC code EMC3-EIRENE ${ }^{[1,2]}$ is developed to enable plasma edge simulations in complex (3D) geometries as present in helical devices such as W7-X and W7-AS. It has also been applied to several tokamaks, including the International Thermonuclear Experimental Reactor (ITER). ${ }^{[2]}$

The EMC3-EIRENE code system is solved by iterating both codes alternatingly until the relative change in the solution is typically $\leq 1 \%$. It is assumed that this value is a measure of the statistical error on the solution. ${ }^{\left[{ }^{3]}\right.}$ In order to investigate the convergence and accuracy for non-linear MC codes, an MC code is set up to solve a simplified non-linear 1D kinetic equation for neutral particles evoking an application of non-linear neutral transport in the proximity of a pumping duct.

We define the numerical error contributions in iteratively solved MC codes similar to those in coupled finite-volume/Monte Carlo (FV-MC) codes, ${ }^{[4]}$ where the numerical error is separated into four contributions. Discretization error is present because approximations are made by using a finite number of grid cells. Convergence error exists when successive iterations result in different results. Finite sampling bias is defined as the deterministic error due to the finite number of MC particles per iteration. Finally, statistical error is the stochastic error due to the finite number of MC particles and has a probability distribution with zero mean.

For this study, we distinguish three different iteration schemes: Correlated Sampling (CS), Random Noise (RN), and Robbins Monro (RM). With CS, the same seeds are used in each iteration to induce correlation of the particle trajectories in successive 
iteration steps. This way, the relative change can eventually decrease to machine accuracy. With RN, different seeds are used each iteration and, therefore, the relative change will keep fluctuating as a result of the interplay between the statistical approach for the simulated trajectories and the iterative procedure. With RM, the current iteration makes use of the average of all previously calculated iterations (with different seeds each iteration) and, as a consequence, the relative change will decrease slowly.

The next section gives more details about the case and its implementation. Subsequently, the discretization error, which is independent of the iterative procedure, will be discussed. Sections 3-5 discuss the remaining error contributions for, respectively, RN, CS, and RM.

\section{2 | CASE DESCRIPTION AND IMPLEMENTATION}

This simple 1D test case describes the neutrals near a pump with the following kinetic transport equation:

$$
\int \Sigma_{s} \Phi\left(x, \vec{\omega}^{\prime}\right) C\left(x, \vec{\omega}^{\prime} \rightarrow \vec{\omega}\right) d \vec{\omega}^{\prime}-\vec{\omega} \cdot \vec{e}_{x} \frac{\partial \Phi}{\partial x}+\Sigma_{s} \Phi=0,
$$

where $\Phi=v f(x, \vec{\omega})$ represents the neutral transport flux. Neutrals move mono-energetically $(T=40 \mathrm{eV})$ with speed $v$ in two directions $\vec{\omega}= \pm \vec{e}_{x}$, with $\vec{e}_{x}$ the unit vector towards the target in the poloidal direction. Only neutral-neutral interactions with macroscopic cross-section $\Sigma_{\mathrm{s}}$ are modelled. They are implemented using the BGK (Bhatnagar-Gross-Krook) approximation, where the neutral density $n_{n}(x)=\sum_{\vec{\omega}} \frac{\Phi^{i-1}(x, \vec{\omega})}{v}$ of the previous iteration $i-1$ is used as background for collisions in the next iteration. ${ }^{[5,6]}$ After a collision, the particle has a 50\% chance of changing its direction: $C\left(x, \vec{\omega}^{\prime} \rightarrow \vec{\omega}\right)=0.5 \delta\left(\vec{\omega}-\vec{\omega}^{\prime}\right)+$ $0.5 \delta\left(\vec{\omega}+\vec{\omega}^{\prime}\right)$, with $\delta$ the Dirac delta function. The cross-section is chosen 100 times larger than in a realistic simulation to increase the collision rate and obtain a clearly non-constant neutral density profile. At $x=0 \mathrm{~m}, 80 \%$ of the incoming neutrals are pumped away. At $x=0.1 \mathrm{~m}$, all neutrals are reflected and there is an incoming neutral flux. In the MC code, several particle trajectories are followed, where random numbers determine the exact flight. Track length estimators are used to evaluate the neutral density $n_{\mathrm{n}}$. More generally, the solution is indicated with the symbol $\phi$. To improve convergence of the solution $\phi$, a relaxation factor $r_{\mathrm{f}}$ is used as follows before continuing to the next iteration $i+1$ :

$$
\phi^{i+1}=\left(1-r_{\mathrm{f}}\right) \phi^{i}+r_{\mathrm{f}} \phi^{i+1} .
$$

This equation is implemented not only with an MC method but also with a finite volume (FV) method to obtain reference solutions. An approximate exact solution $\phi^{\mathrm{EX}}$ is obtained by making the FV code converge with 8000 grid cells. In addition, reference solutions $\phi^{\mathrm{FV}}$ are constructed that approximate the MC solution with an infinite number of MC particles on a specific, coarse grid. To achieve this, the behaviour of the MC code is mimicked with the FV code: the background variables from the coarse grid are kept equal in the fine grid, which results in piece-wise constant profiles on the fine grid. To return to the coarse grid, the densities obtained on the fine grid are averaged over the coarse grid cells. With these solutions, the influence of the number of MC particles can be analysed without interference of the discretization error.

To monitor the change in the iterative solution during the MC simulations, we use the following formula ${ }^{[3]}$ :

$$
C_{\mathrm{r}}[\%]=\frac{\sum_{x}\left|\phi_{x}^{i-1}-\phi_{x}^{i}\right| V_{x}}{\sum_{x}\left(\phi_{x}^{i-1}+\phi_{x}^{i}\right) V_{x}} \cdot 100,
$$

where $x$ and $i$ are, respectively, the cell index and iteration number, and $V_{x}$ is the cell volume. When the code converges, $C_{\mathrm{r}}$ ideally becomes zero. In this paper, the error will be expressed similarly as

$$
\varepsilon[\%]=\frac{\sum_{x}\left|\phi_{x}^{\mathrm{ref}}-\phi_{x}^{i}\right| V_{x}}{2 \sum_{x} \phi_{x}^{\mathrm{ref}} V_{x}} \cdot 100,
$$

with $\phi^{\text {ref }}$ a reference solution, which, depending on the examined error, can be $\phi^{\mathrm{EX}}$ or $\phi^{\mathrm{FV}}$, or the converged solution in a CS simulation.

In the next sections, we discuss numerical errors for several iterative procedures: RN, CS, and RM. With RN, different seeds are used in each iteration. Therefore, because of the statistical noise, the relative change $C_{r}$ cannot decrease to machine accuracy and remains fluctuating around a constant level. With CS, where the same seeds are used in each iteration, strict convergence of $C_{\mathrm{r}}$ can be reached if the particle trajectories remain correlated between the iterations. With RM, averaging is used during the simulation, and $C_{\mathrm{r}}$ can slowly converge. The finite sampling bias, convergence error, and statistical error are highly influenced by the chosen iterative procedure. The discretization error, on the other hand, is independent of the iterative procedure. Therefore, we start with discussing the discretization error before giving more details on the iterative procedures. 


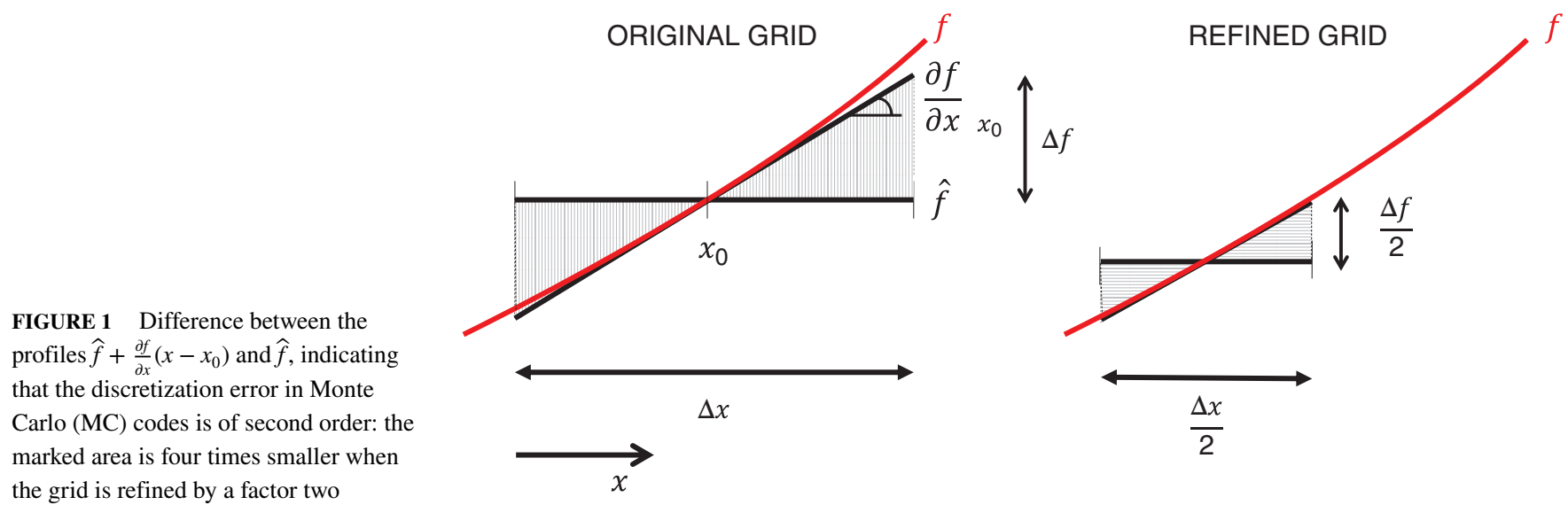

FIGURE 2 Second-order convergence of the discretization error demonstrated in numerical experiments for the reference solutions $\phi^{\mathrm{FV}}$ and Random Noise (RN) simulations

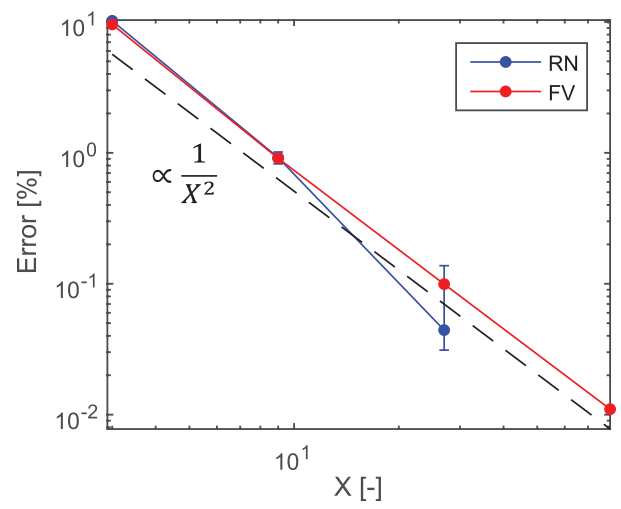

\section{3 | DISCRETIZATION ERROR}

Discretization error originates from approximations related to the finite number of grid cells. In an MC code, the value of the scored variables is kept constant in each cell: when the scoring tallies, one value is stored for the whole cell. Moreover, during the particle trajectories, cross-sections are calculated based on the background densities obtained in the previous iteration and are assumed to be constant in each cell. In this case, the MC code approximates the variables with a piece-wise constant function.

The magnitude of the discretization error is proportional to the difference between the approximated profile $\hat{f}$ (piece-wise constant) and the profile that would have been obtained without this approximation, which we call the reference profile $f$. The constant value that approximates the profile in a cell is the mean value of the reference profile. We expect that the discretization error is proportional to the integral of the absolute value of the difference between these two profiles:

$$
\varepsilon_{d} \propto \int|f-\widehat{f}| d x
$$

Applying a Taylor approximation ${ }^{[7]}$ in each cell, and keeping only the first term which is asymptotically dominant, the function $f=\widehat{f}+\left.\frac{\partial f}{\partial x}\right|_{x_{0}}\left(x-x_{0}\right)+\cdots$ is obtained. The integral is now easily calculated as

$$
\left.\left.\varepsilon_{d} \propto \frac{\partial f}{\partial x}\right|_{x 0} \int_{x_{0}-\Delta x / 2}^{x_{0}+\Delta x / 2}\left(x-x_{0}\right) d x \propto \frac{\partial f}{\partial x}\right|_{x_{0}}\left(x-x_{0}\right) \Delta x \propto \frac{(\Delta x)^{2}}{2}
$$

This indicates that the discretization error has an asymptotic order of convergence of two, as seen in Figure 1.

Figure 2 is obtained from simulations on different grid sizes and shows that the error reduction rate is indeed of second order. The error is calculated using the reference solution $\phi^{\mathrm{EX}}$ obtained with a very fine grid ( $\phi^{\mathrm{ref}}$ in Equation 4 is $\phi^{\mathrm{EX}}$ ). The error on the reference solutions $\phi^{\mathrm{FV}}$, which are only affected by the discretization, is indicated in blue. The red lines have been obtained with RN, where the numbers of particles and iterations have been chosen sufficiently large to obtain a dominant discretization error. The error bars indicate the estimated magnitude of the statistical error and bias. 

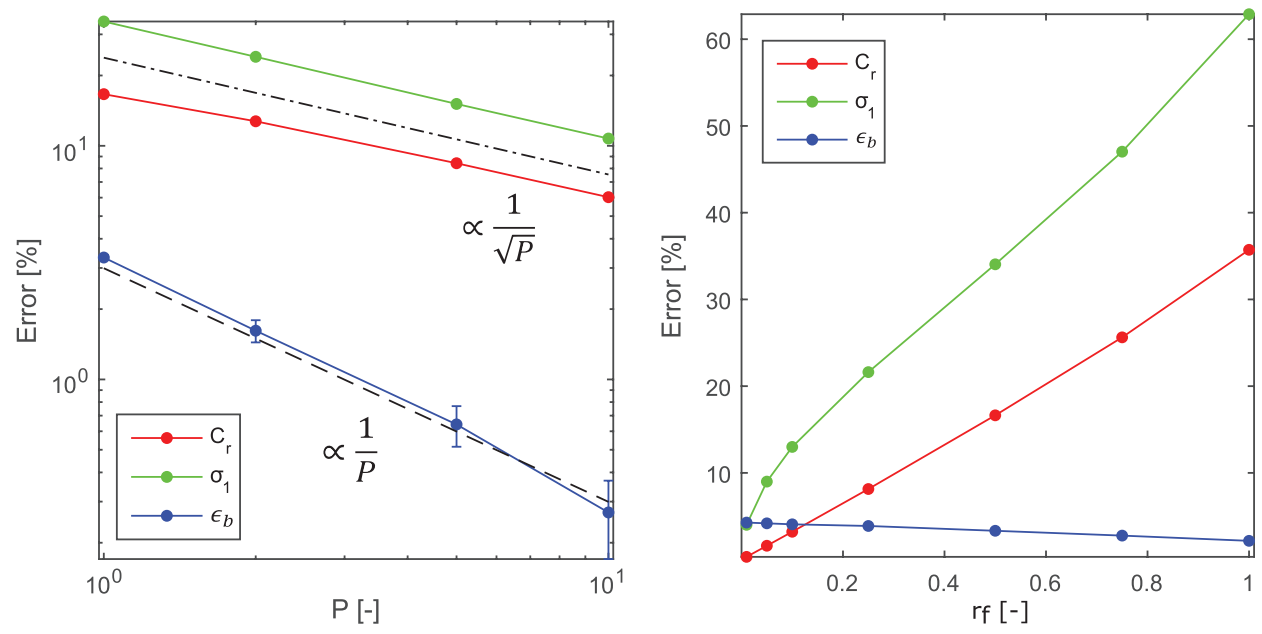

FIGURE 3 Relative change $C_{\mathrm{r}}$ (red), bias $\varepsilon_{\mathrm{b}}$ (blue), and standard deviation $\sigma_{1}$ (green) as a function of the number of Monte Carlo (MC) particles per iteration $P$ (left, with $r_{\mathrm{f}}=0.5$ ) and the relaxation factor $r_{\mathrm{f}}$ (right, with $P=1$ )

\section{I RANDOM NOISE}

In RN simulations, different seeds are used during each iteration. Because of the changing statistics, the relative change cannot decrease to machine accuracy, but remains fluctuating. However, after an initial transient phase, the variables remain statistically stationary. To construct the solution of an RN simulation, a post-processed average is taken over the statistically stationary iterations.

Besides the discretization error, there is a bias as well as a statistical error present on the solution. The bias exists because of non-linearities in the model and takes into account the convergence error and the finite sampling bias. It decreases rapidly with the number of MC particles: $\varepsilon_{b} \propto(1 / P)$, as shown in Figure 3 (left). The statistical error has a probability distribution with mean 0 and standard deviation $\sigma$. By averaging, the statistical error on the solution is governed by $\sigma=\sigma_{1} / \sqrt{I / T}$, with $\sigma_{1}$ the standard deviation of the iterations, $I$ the number of iterations over which is averaged, and $T$ the correlation time which takes into account that consecutive iterations are not independent of each other. Figure 3 shows that the standard deviation of the iterations decreases with the number of MC particles per iteration: $\sigma_{1} \propto 1 / \sqrt{ } P$. The relative change follows the same reduction rate: $C_{\mathrm{r}} \propto 1 / \sqrt{ } P$, as indicated in Figure 3 .

The relaxation factor $r_{\mathrm{f}}$ influences the magnitude of the errors, as shown in Figure 3 (right). Because consecutive iterations will differ less with a smaller relaxation factor, the relative change $C_{\mathrm{r}}$ decreases. Consecutive iterations will also fluctuate less around the bias, as indicated by the decreasing standard deviation $\sigma_{1}$. The correlation time, however, which is a measure of the dependence between consecutive iterations, will increase such that the statistical error on an averaged solution $\sigma\left(=\sigma_{1} / \sqrt{I / T}\right)$ remains approximately constant for a specified number of iterations $I$. It is also observed that the bias $\varepsilon_{\mathrm{b}}$ increases with a decreasing relaxation factor.

Because similar reduction rates are retrieved, the relative change $C_{\mathrm{r}}$ can be a good indicator for the standard deviation of the iterations $\sigma_{1}$, though it is not a measure for the statistical error $\sigma$ of the solution itself. However, for a reliable estimate, it is more accurate to calculate $\sigma_{1}$ as the sample standard deviation directly from the iterations. Moreover, $C_{\mathrm{r}}$ provides information only about the statistical error, while the discretization error and the bias should not be neglected. They can be estimated by comparing solutions with different grid sizes and particles per iteration, analogous to the procedure in FV-MC codes. ${ }^{[4]}$

Finally, it is noted that, in practice, this iterative procedure is often used without averaging. However, averaging has the potential to speed up the simulations significantly through reducing the number of particles per iteration without increasing the statistical error. This is especially beneficial if the initial transient phase is long. With the FV-MC code B2-EIRENE, an order of magnitude speed-up could be obtained by introducing averaging. ${ }^{[4,8]}$

\section{5 | CORRELATED SAMPLING}

With CS, the same seeds are used for each iteration with the aim of improving the convergence of the solution. Convergence is meant in terms of the relative change tending to zero with an increasing number of iterations. Taking the same seeds is, however, not always sufficient. Particle trajectories can, for example, lose correlation as a result of discontinuities in the discretized background profiles. ${ }^{[9]}$ Baeten ${ }^{[9]}$ introduced two ways to decrease the presence of discontinuities and thereby increase the probability of achieving convergence. First, to eliminate discontinuities coming from the piece-wise constant approximation of the cross-sections, a linear interpolation can be used during the simulation. Second, to avoid large differences in particle weight between particles reaching the pump (where the particle weight is significantly reduced) and particles changing direction shortly 
FIGURE 4 Error contributions with correlated sampling (CS). The left figure shows the convergence error in function of the relative change for several relaxation factors $(0.2,0.5,1.0)$. The right figure shows the bias $\varepsilon_{\mathrm{b}}$ and the standard deviation $\sigma_{1}$ for both CS (full lines) and Random Noise (RN) (dotted lines)
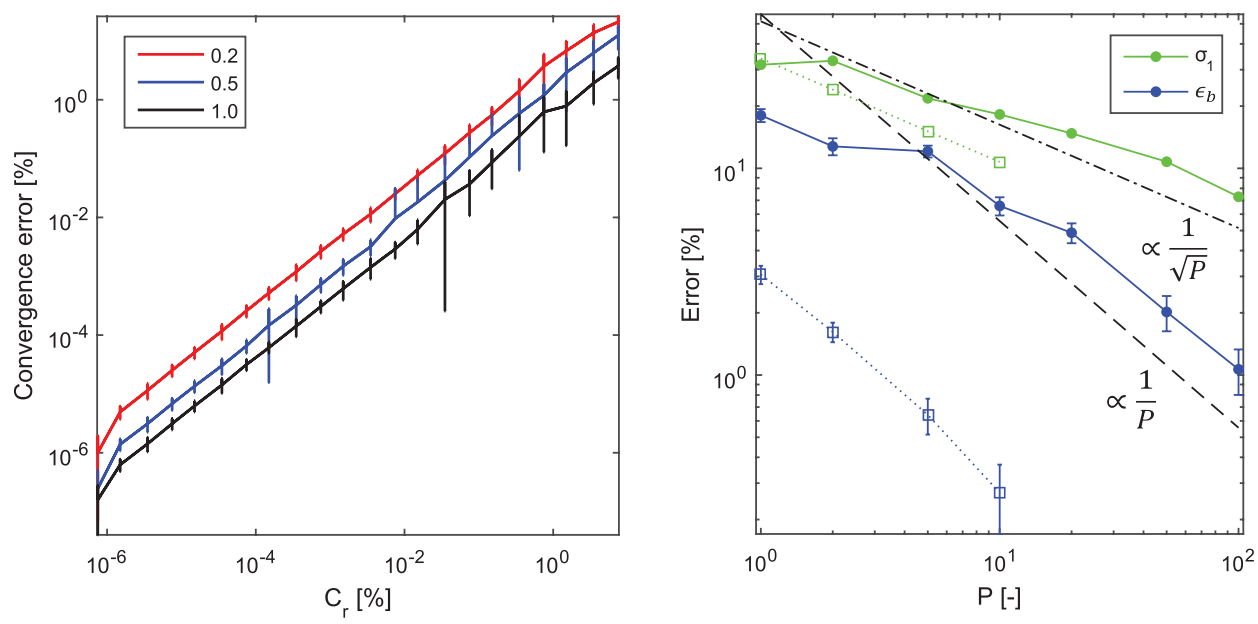

before reaching the pump (with only a small reduction in weight), a so-called continuate boundary can be implemented. A linear profile for weight reduction is imposed from a short distance before the pump to the pump itself. That way, particles colliding shortly before the pump and immediately after the pump will experience a similar reduction in weight.

These measures have an influence on the accuracy of the solution. Linear interpolation of the cross-section has only a small effect, which is considered to be positive since a linear profile is a more accurate representation than a piece-wise constant profile. The continuate boundary approach, however, can introduce a significant deterministic error on the solution. Moreover, this error is not limited to the boundaries since particles can travel across the whole domain. Because of this additional error on the solution, the continuate boundary should be used only when achieving convergence is more important than accuracy and, preferably, only for those particle trajectories that experience decorrelation due to the weight discontinuity at the boundary.

The probability of convergence is also influenced by many simulation and model parameters. With longer particle trajectories, for example, the trajectories also need to remain correlated for longer, and the probability of convergence decreases. With less collisionality and thus smaller Knudsen numbers, fewer collisions happen and the probability of convergence increases. Another cause of decorrelation of particle trajectories is the changing neutral background, which differs during each iteration. The probability of achieving convergence is, therefore, increased by decreasing the relaxation factor.

Although achieving convergence is essential with CS, the convergence error only needs to be negligible compared to the other error contributions, which was easily achieved in this simple model using the above methods. Depending on the relaxation factor, a different value of the relative change is required to obtain a specific convergence error. This is shown in Figure 4 (left), which was obtained from 100 converged runs with $P=100$ for relaxation factors $0.2,0.5$, and 1 . The error bars indicate the standard deviation of the convergence error, which decreases with increasing number of MC particles $P$.

Figure 4 (right) shows the standard deviation $\sigma_{1}$ and the finite sampling bias $\varepsilon_{\mathrm{b}}$ with both CS (full lines) and RN (dotted lines). The expected error reduction rates $\sigma_{1} \propto 1 / \sqrt{ } P$ and $\varepsilon_{\mathrm{b}} \propto 1 / P$ are asymptotically retrieved (from $\approx P=20$ ). In contrast with RN, $\sigma_{1}$ and $\varepsilon_{\mathrm{b}}$ with CS are independent of the relaxation factor. As expected from similar results with FV-MC codes, the standard deviation $\sigma_{1}$ and, especially, the finite sampling bias $\varepsilon_{\mathrm{b}}$ are much larger with CS than with RN for the same number of particles per iteration $P$. This also makes the computational time required for an accurate result to be much larger than with $\mathrm{RN}$ (as demonstrated in ref. ${ }^{[4]}$ ). We, therefore, consider CS to be a less efficient iterative procedure than RN. CS can, however, be useful for other purposes such as, for example, the calculation of sensitivities.

\section{6 | ROBBINS MONRO}

In $\mathrm{RM}^{[10]}$ the background profile used in each new iteration $I$ is the average of all previously calculated profiles. This average is also taken as the solution. In practice, this can be achieved using an adaptive relaxation factor $\alpha(I)=1 / I .{ }^{[11]}$ Because of the decreasing relaxation factor, it is expected that the relative change $C_{\mathrm{r}}$ also keeps decreasing. Indeed, Figure 5 (left) shows the following reduction rate: $C_{\mathrm{r}} \propto 1 / I$.

The full lines in Figure 5 show the evolution of the obtained error for both RM and RN in a simulation with $P=1$. Initially, the total error decreases because of the averaging procedure, which decreases the statistical error in both iterative procedures. After approximately $10^{3}-10^{4}$ iterations, the deterministic error becomes dominant and the total error remains almost constant. While the initial error decrease is similar for both iterative procedures (approximately $\propto 1 / \sqrt{ } I$ ), the deterministic error is much larger with RM. This can be attributed to the continuously decreasing relaxation factor in the RM procedure. Returning to Figure 3, it is clear that the deterministic error with RM is equivalent to the bias with RN in the limit of $r_{f}$ going to zero. The relative 


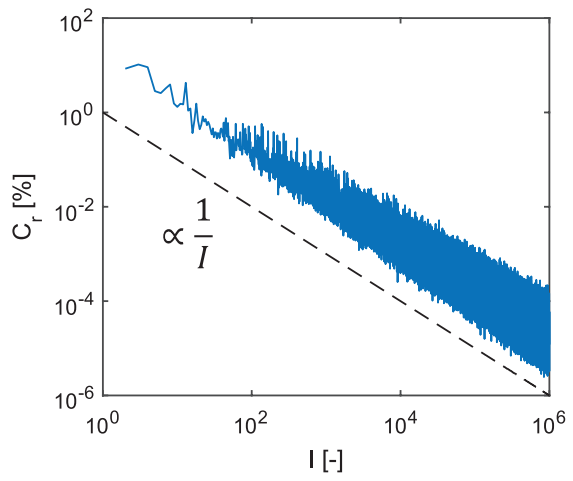

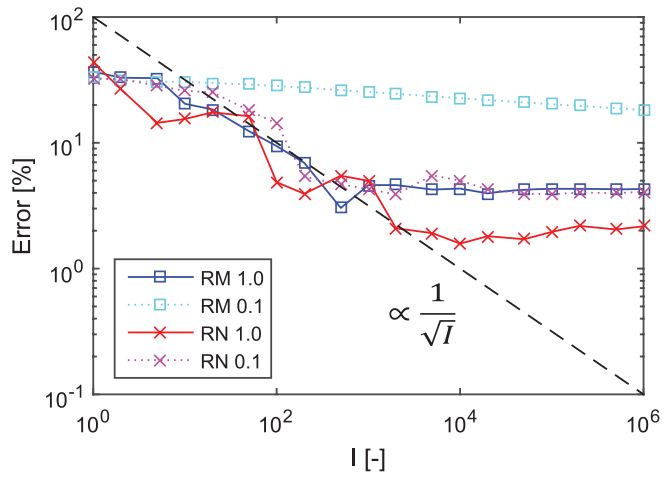

FIGURE 5 Relative change $C_{\mathrm{r}}$ (left) and the total error (right) in function of the number of iterations $I$

change $C_{\mathrm{r}}$ is clearly not a good error indicator for this procedure. While $C_{\mathrm{r}}$ keeps decreasing with the number of iterations, the error does not. In addition, the initial reduction rate of the error (approximately $\propto 1 / \sqrt{ } I$ ) is different from that of $C_{\mathrm{r}}(\propto 1 / I)$.

In this simplified case, consecutive iterations have a relatively small dependence on each other with a correlation time of only a few iterations. However, in more complex codes and especially when multiple codes are coupled, the correlation between iterations is expected to be much larger. This can be imitated by choosing a smaller relaxation factor $r_{\mathrm{f}}$ in both RN and RM. In that case, the adaptive relaxation factor with RM becomes $\alpha(I)=r_{\mathrm{f}} / \mathrm{I}$. The dotted lines in Figure 5 show the evolution of the error for RN and RM with a relaxation factor of 0.1 . The error with RN still decreases relatively fast and remains constant at a bias expected at this relaxation factor. With RM, however, the initial decrease in error is extremely slow. This is in contrast with the relative change $C_{\mathrm{r}}$, which still exhibits $1 / \mathrm{I}$ behaviour and can thus be very misleading as an error indicator. In addition, it is challenging to obtain a reliable total error indication for RM without a reference solution. Because RN provides a more accurate result in general, it is recommended to use this iterative procedure rather than RM.

\section{7 | CONCLUSION}

We discussed the accuracy and convergence of an iteratively solved MC code for three iterative procedures: RN, CS, and RM. We divided the total numerical error in different contributions based on their origin (discretization, finite number of MC particles, convergence) and type (deterministic, stochastic). These definitions have also been used for error assessment in FV-MC codes. ${ }^{[4]}$ In general, similar conclusions can be drawn. RN (with averaging) is generally the most efficient iterative procedure, realizing a small bias with a relatively small number of MC particles per iteration. With CS, much larger numbers of MC particles per iteration are required to obtain accurate results. Moreover, strict convergence can be hard to achieve in practice. Several methods have been discussed to improve the probability of reaching a sufficiently small convergence error. Although the relative change in RM seems to indicate convergence, the error does not keep decreasing. Moreover, it is unfeasible to evaluate the error without a reference solution.

Usually, iteratively solved MC codes like EMC3-EIRENE are solved with RN. However, post-processing averaging to decrease the statistical error is not commonly used. The typical practice to use the relative change as error indicator is easy but insufficient as an estimate for the total numerical error, as it does not take into account the deterministic errors. The discretization error and bias can be estimated by comparing solutions with, respectively, different grid sizes and different numbers of MC particles per iteration. Instead of using the relative change, the statistical error can be estimated more accurately based on the sample standard deviation.

\section{AKNOWLEDGEMENT}

This work was sponsored by Flanders Innovation and Entrepreneurship (IWT.141064).

\section{REFERENCES}

[1] Y. Feng, F. Sardei, J. Klissinger, J. Nucl. Mater. 1999, 266-269, 812.

[2] Y. Feng, H. Frerichs, M. Kobayashi, A. Bader, F. Effenberg, D. Harting, H. Hoelbe, J. Huang, G. Kawamura, J. D. Lore, T. Lunt, D. Reiter, O. Schmitz, D. Sharma, Contrib. Plasma Phys. 2014, 54(4-6), 426.

[3] M. Kobayash, Y. Feng, F. Sardei, D. Reiter, D. Reiser, and K. H. Finken, Contrib. Plasma Phys. 2004, 44, 1.

[4] K. Ghoos, W. Dekeyser, G. Samaey, P. Börner, M. Baelmans, J. Comput. Phys. 2016, 322, 162.

[5] D Reiter, The EIRENE Code User Manual 2017.

[6] D. Reiter, C. May, M. Baelmans, P. Börner, J. Nucl. Mater. 1997, 241-243, 342.

[7] J. Ferziger, M. Peric, Computational Methods for Fluid Dynamics, 3rd ed., Springer-Verlag, Berlin Heidelberg 2002. 
[8] M. Baelmans, P. Börner, K. Ghoos, Nucl. Mater. Energy 2016, 12(2017), 858-863. http://dx.doi.org/10.1016/j.nme.2016.10.028.

[9] M. Baeten, B. Mortier, M. Baelmans, G. Samaey. Proc.Int. Conf. Mathematics and Computational Methods Applied to Nuclear Science and Engineering, Jeju, Korea.

[10] H. Robbins, S. Monro, Ann. Math. Stat. 1951, 22, 400.

[11] H. G. Frerichs, Three-Dimensional Plasma Transport in Open Chaotic Magnetic Fields: A Computational Assessment for Tokamak Edge Layers, RWTH Aachen, FZJ Julich, Julich, Germany 2010.

How to cite this article: Ghoos K, Samaey G, Baelmans M. Accuracy and convergence of iteratively solved Monte Carlo codes for simulations in the plasma edge of nuclear fusion reactors. Contributions to Plasma Physics 2018;58:652-658. https://doi.org/10.1002/ctpp.201700181 